\title{
Review \\ Single-Cell Microgels: Technology, Challenges, and Applications
}

Tom Kamperman, ${ }^{1, @}$ Marcel Karperien, ${ }^{1, @}$ Séverine Le Gac, ${ }^{2, @}$ and Jeroen Leijten ${ }^{1, *, @ ~}$

Single-cell-laden microgels effectively act as the engineered counterpart of the smallest living building block of life: a cell within its pericellular matrix. Recent breakthroughs have enabled the encapsulation of single cells in sub-100- $\mu \mathrm{m}$ microgels to provide physiologically relevant microniches with minimal mass transport limitations and favorable pharmacokinetic properties. Single-cellladen microgels offer additional unprecedented advantages, including facile manipulation, culture, and analysis of individual cell within 3D microenvironments. Therefore, single-cell microgel technology is expected to be instrumental in many life science applications, including pharmacological screenings, regenerative medicine, and fundamental biological research. In this review, we discuss the latest trends, technical challenges, and breakthroughs, and present our vision of the future of single-cell microgel technology and its applications.

\section{Size Matters: Advantages of Hydrogel Miniaturization}

The field of biotechnology is rapidly evolving towards single-cell applications. In recent years, myriad tools have been developed for in-depth single-cell experimentation. These tools have almost exclusively relied on nonphysiological 2D substrates and, more recently, on droplet microfluidics-based formats, which is acknowledged as a promising platform for single-cell experimentation [1,2]. The introduction of hydrogels (see Glossary) into these platforms has the potential to provide individual cells with a more physiologically relevant 3D microenvironment (Box 1). To make hydrogel-based technologies readily compatible with high-resolution single-cell experimentation as well as in vivo applications, there is an ongoing trend of miniaturizing hydrogel constructs down to the micrometer scale, to yield microgels. Encapsulation of single cells in microgels effectively creates high-resolution 3D microniches that provide exquisite control over the culture and analysis of cells in vitro, as well as the fate of cellbased therapies in vivo.

The first hydrogel-based 3D cell constructs relied on the encapsulation of multiple cells in a bulk biomaterial [3]. Although such relatively large (typically $>1 \mathrm{~mm}$ ) constructs are comparatively easy to manufacture and handle, they often result in biased experimental readouts and poor therapeutic effects. For example, due to mass transport limitations, the encapsulated cells experience different levels of nutrients and waste products, and even hypoxic conditions, depending on the position of the cells within the construct. Hypoxia-induced cell death can have a significant role in hydrogel constructs as small as a few hundred micrometers [4]. Furthermore, millimeter-sized hydrogel constructs are associated with significantly higher levels of fibrotic capsule formation when implanted in vivo compared with their micrometer-sized counterparts [5]. For instance, by implanting agarose microgels with diameters ranging from 90 to $925 \mu \mathrm{m}$ in mice and rats, Kawakami and coworkers found that the microgel size correlated with the amount of tissue overgrowth, and that microgels smaller than $100 \mu \mathrm{m}$ caused the least
Highlights

Several recent technological breakthroughs have facilitated and improved the manufacturing of single-cell-laden microgels. In particular, microfluidic technologies have enabled the highyield and long-term encapsulation of individual cells in microgels.

Encapsulating individual cells into micrometer-sized hydrogels (i.e., microgels) provides user-defined $3 \mathrm{D}$ culture conditions that can be leveraged to advance single-cell analysis platforms, cell-based tissue engineering, and regenerative medicine applications.

Early adopters are currently developing and implementing various highthroughput micromanufacturing techniques that will empower the clinical and industrial translation of singlecell-laden microgels as versatile highresolution 3D cellular microniches for numerous applications.

${ }^{1}$ Department of Developmental BioEngineering, MIRA Institute for Biomedical Technology and Technical Medicine, University of Twente, Drienerlolaan 5, 7522NB Enschede, The Netherlands ${ }^{2}$ Applied Microfluidics for BioEngineering Research, MESA+ Institute for Nanotechnology, MIRA Institute for Biomedical Engineering and Technical Medicine, University of Twente, Drienerlolaan 5, 7522NB Enschede, The Netherlands ${ }^{\circledR}$ Twitter: @DBE_MIRA, @UTwente, @utwenteEN, @DBE_MIRA

${ }^{*}$ Correspondence:

j.c.h.leijten@utwente.nl (J. Leijten). 


\section{Trends in Biotechnology}

CellPress

REVIEWS

\section{Box 1. Shifting from 2D Substrates to 3D Microenvironments}

Since the inception of in vitro experimentation, cells have been cultured on 2D substrates. However, the native microenvironment of almost all cells is 3D. Consequently, 2D substrates do not fully recapitulate the in vivo situation because they impose nonphysiological stimuli, such as unnatural and anisotropic chemical and physical signals, on cells (Figure I). Anisotropic stimulation of cells can cause phenotypical cellular changes, which may result in false-positive and false-negative cellular responses. To emulate the native cellular microenvironment and restore natural cellular functions and behavior, more advanced 3D cell experimentation platforms are required, which is notably achieved by encapsulating cells in hydrogels. Hydrogels structurally resemble the native extracellular matrix and can be biochemically and biophysically modified to further mimic the in vivo microenvironment. An overview of the most important differences between 2D and 3D culture systems with respect to biochemical and biophysical stimuli was provided by Baker and Chen [86].

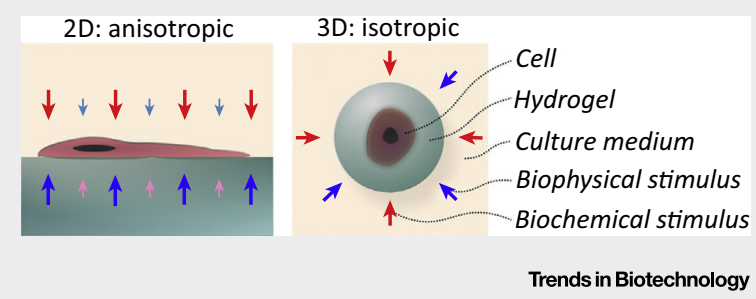

Figure I. 2D versus 3D Cell Culture. 2D substrates present anisotropic biochemical and biophysical stimuli to cells. By sharp contrast, 3D environments, such as single-cell-laden microgels, stimulate cells in an isotropic manner. The size and intensity of the arrows correspond to the strength of the stimuli, with larger and darker arrows indicating stronger stimuli.

amount of fibrosis [6]. In addition, an increased construct size is not favorable for highthroughput analysis (e.g., due to visualization limitations resulting from both light scattering and penetration depth) [7]. Although these limitations can be addressed via physical sectioning (i.e., microtomy), sample processing is costly, not standardized, and labor intensive, which greatly reduces the analytical throughput. All these limitations have driven the miniaturization of cell-laden hydrogel constructs, which has led to the encapsulation of individual cells in microgels.

Due to their significantly reduced size, microgels offer higher surface:volume ratios, reduced diffusion (limitations), and direct compatibility with many standard microscopy techniques compared with conventional larger hydrogels. Reducing the size of a hydrogel to the micrometer scale has, for example, been demonstrated to improve insulin release kinetics from multicell-laden [8] as well as single-cell-laden microgels [9]. Furthermore, for in vivo applications, the microgel size can be used to control the in vivo biodistribution, and thereby to regulate the location of encapsulated compounds. For example, intravenously injected single-stem-cellladen microgels of $\sim 30 \mu \mathrm{m}$ were recently found to accumulate in the lung capillary beds of mice [10]. By contrast, intravenously injected microgels that are larger than $\sim 50 \mu \mathrm{m}$ typically get physically trapped and, thus, accumulate in blood vessels of downstream tissues, with the inherent risk of tissue infarction. Altogether, the microgel size has a dominant effect on several pharmacokinetic properties that determine the physiological responses following implantation, as summarized in Figure 1.

Miniaturizing single-cell-laden microgels is also advantageous for tissue-engineering applications. For example, the size of the microgel is correlated to the maximum cell concentration of the construct. In fact, the maximum packing density of spheres dictates that single-cell-laden microgels smaller than $50 \mu \mathrm{m}$ are required to reach cell densities of $\geq 10^{6}$ cells per $\mathrm{cm}^{3}$ [11], which is the physiologically required minimum for engineering many tissues [12-14]. Furthermore, downsizing single-cell-laden microgels provides an opportunity to optimize engineered

\section{Glossary}

Air-induced spraying: a droplet production method based on the atomization of liquid using a coaxial air flow.

Antibiogram: the summarized result of testing pathogenic sensitivity towards particular antimicrobials.

Bio-orthogonal: with no interference or interaction with native biological and biochemical processes.

Chelator: a binding agent that can sequester metal ions.

Cytokines: secreted small proteins that chemically facilitate the communication between cells. Dripping: the droplet-forming process where liquid pinches of directly from the nozzle.

Droplet microfluidics: fluidic platforms with characteristic length scales of 1-1000 $\mu \mathrm{m}$ that are used to produce, guide, manipulate, and contain emulsified (i.e., droplets) liquids.

Electrospraying: a droplet production method where electrostatic forces are used to disrupt a liquid surface at a nozzle to form a continuous stream of charged droplets.

Extracellular: outside of a cell. Fibrotic capsule: a layer of fibrous tissue that is formed around implanted materials as a result of inflammation against the foreign material.

Flow cytometry: laser- or impedance-based method to analyze particles (e.g., cells) in a focused fluid stream.

Hydrogel: a water-swollen crosslinked polymer network. Inertial focusing: the migration of particles across streamlines to deterministic equilibrium positions within a confined flow.

Infarction: the death of tissue due to inadequate blood supply. Inkjet: a droplet production method that relies on a sudden push of the liquid interface contained by a nozzle, resulting in the ejection of an elongated jet that subsequently contracts into a droplet.

Interpenetrating: interlaced and/or entangled, but, in this context, not covalently bound.

Jet: a droplet formation process where liquid exits the nozzle as a continuous stream and pinches of 


\section{Trends in Biotechnology}

\section{CellPress \\ REVIEWS}

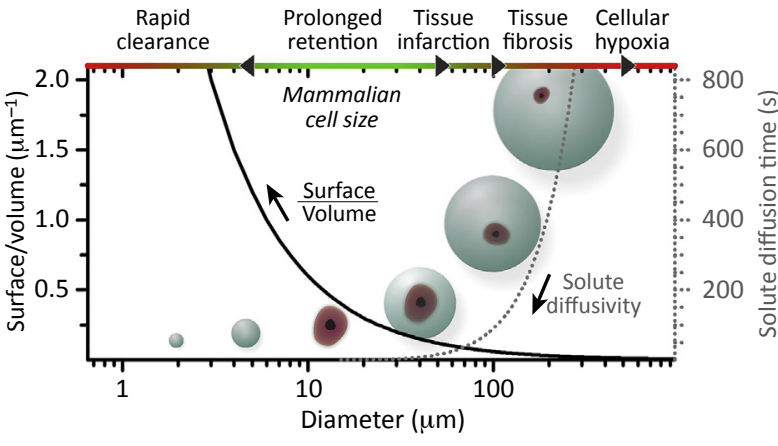

Trends in Biotechnology
Figure 1. Influence of the Microgel Size on Pharmacokinetics and Physiological Responses. Reducing the single-cell microgel diameter $\left(D_{\text {gell }}\right)$ correlates to an increasing surface:volume ratio $\left(6 / D_{\text {gell }}\right)$ and a decreasing solute diffusion time $\left[\left(D_{\text {gel }}-D_{\text {cell }}\right)^{2} / 2 D\right.$, with cell diameter $D_{c e l l}=15 \mu \mathrm{m}$ and diffusion coefficient $D=10 \mu \mathrm{m}^{2} / \mathrm{s}$ ]. Furthermore, the size of the (single-cell) microgel determines its in vivo fate and physiological effect. Microgels larger than $5 \mu \mathrm{m}$ are characterized by significantly increased retention times, because their rapid clearance from the injection site is prevented $[89,90]$. Increasing the microgel size to above $50 \mu \mathrm{m}$ may cause tissue infarctions due to vessel embolization, tissue fibrosis (i.e., above $100 \mu \mathrm{m}$ ), and eventually hypoxia-induced death of cells encapsulated in microgels larger than a few hundred micrometers.

tissue constructs by exclusively presenting bioactive moieties (e.g., growth factors) in the direct vicinity of cells [11]. Leaving the bulk of the extracellular matrix void of exogenous growth factors would allow for a significant reduction in the required growth factor payload in engineered tissues, thereby potentially obviating the adverse effects associated with supraphysiological growth factor administration, which include inflammation, vascular leakage, edema, aberrant angiogenesis, and even cancer $[15,16]$.

\section{Composition of the Single-Cell Microgel}

The single-cell-laden microgel customarily comprises a hydrogel matrix resembling the pericellular matrix, which encapsulates a cell and, if desired, is complemented with soluble factors (e.g., cytokines). The hydrogel matrix is a water-swollen polymer network that acts as the direct physical microenvironment for the encapsulated cell. By engineering this microenvironment to provide well-balanced biochemical (i.e., bioactive solutes and polymer functionalization) and biophysical stimuli (i.e., mesh size, stiffness, stress relaxation, degradation, and polymer type), the single-cell-laden microgel can survive and function on its own. Indeed, the single-cell-laden microgel effectively acts as the engineered counterpart of the smallest living building block of life: a cell within its pericellular matrix (Figure 2).

Traditionally, alginate and agarose have been extensively used for (single) cell microencapsulation. However, these materials lack any (mammalian) cell-specific biological cues or responsiveness. In recent years, the microencapsulation field has started to explore responsive materials to engineer more physiologically relevant cellular microniches. For example, Huck and coworkers pioneered the encapsulation of individual stem cells within collagen, hyaluronic acid, and fibrin microgels [17,18]. Furthermore, Gidrol and coworkers demonstrated that individual epithelial cells encapsulated in Matrigel microgels could develop into clonal multicellular acinar 3D structures [19]. Importantly, all these natural polymers intrinsically contain binding sites, such as the arginine-glycine-aspartate (RGD) sequence that interacts with cell adhesion molecules (CAMs). Additionally, natural polymers can be remodeled and degraded by encapsulated cells through enzymatic cleavage. Others have succeeded in endowing synthetic polymer microgels with cell-adhesive and cleavable moieties, acting as the engineered counterpart of natural microniches. For example, Lutolf and coworkers incorporated biologically several diameters from the nozzle as a result of capillary instability. Jet cutting: a droplet production method based on the physical cutting of a liquid jet using rotating wires.

Microgel: a hydrogel with a size ranging from $1 \mu \mathrm{m}$ to $1000 \mu \mathrm{m}$. Modular bioink: biological ink that contains small modules to enable the one-step fabrication of bottom-up (i. e., modular) biomaterials. Monodisperse: uniform in size, typically characterized by a coefficient of variation (CV: standard deviation/average) of less than $10 \%$. Pericellular: the extracellular domain that is in direct proximity (micrometer range) of the cell.

Pharmacokinetic: the absorption, distribution, metabolism, and excretion of drugs.

Polydisperse: variable in size, typically characterized by a CV greater than $10 \%$.

Rolling circle amplification: a unidirectional nucleic acid replication technique that can rapidly synthesize multiple copies of circular molecules of DNA or RNA.

Stress relaxation: decreasing stress response to a constant strain. Surfactants: compounds that lower the surface tension between two phases.

Trophic mediators: stimulating factors that promote cell survival and functioning.

Vibrating jet: a droplet production method where a uniform oscillation is imposed on a jetted liquid to induce controlled breakup of the jet into monodisperse droplets by dominating the random Rayleigh plateau breakup. 


\section{Trends in Biotechnology}

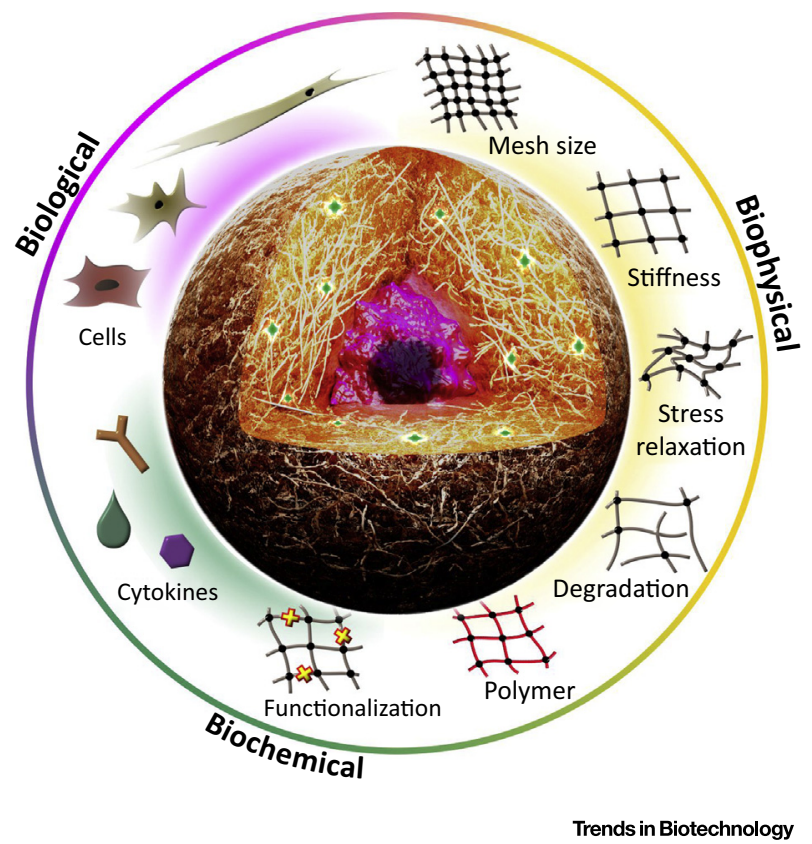

Figure 2. Schematic Overview of the Canonical Composition of Single-Cell-Laden Microgels. The key components include the cell, soluble factors such as cytokines, and the hydrogel matrix, which can be engineered by tuning several parameters, including the mesh size, stiffness, stress relaxation, degradation, polymer type, and functional moieties. All components are categorized by their nature (i.e., biological, biochemical, or biophysical).

active peptide sequences in single-cell-laden bioinert polyethylene glycol (PEG) hydrogel networks to tune cell adhesion (i.e., using RGD peptides) and enzymatic degradation (i.e., using GPQGIWGQ peptides) [20]. The importance of bioactive components in promoting cellular function was underlined in a recent study by Leijten and coworkers, where the lineage commitment of individually encapsulated mesenchymal stem cells was steered from an adipogenic to an osteogenic fate by enriching bioinert dextran microgels with bioactive hyaluronic acid polymer groups [21]. Microgel stiffness has also been demonstrated to exert a profound effect on the differentiation of individual encapsulated stem cells in cell-adhesive and enzymatically degradable microgels [22], which corroborates previous work in bulk hydrogels [23]. Additional functionality comes from tuning the microgel mesh size. For example, single cells have been encapsulated in semipermeable PEG-diacrylate microgels with a molecular weight cut-off of $\sim 100 \mathrm{kDa}$ [11]. This approach has the potential to create immunoprotective microenvironments for individual cells, because it pericellularly blocks the penetration of immunoglobulins ( $\geq 150 \mathrm{kDa}$ ), while still allowing the permeation of nutrients, growth factors, and waste products $(<100 \mathrm{kDa})$.

Although a cornucopia of functional and responsive material modifications has been developed for (bulk) hydrogels [24], material modifications other than cell-adhesive and enzymatically degradable moieties have remained scarce for single-cell-laden microgels. However, considering the large repertoire of bulk hydrogel modifications, we anticipate that the evolution of overly simplistic single-cell-laden microgels into 'smart' constructs will become a dominant theme in single-cell microgel research. Biochemically and biophysically dynamic single-cell microenvironments are of specific interest for the engineering of (stem) cell microniches, because they could emulate the naturally dynamic native cellular microenvironment [25-27]. Specifically, we foresee the formulation of single-cell-laden microgels with bio-orthogonal 


\section{Trends in Biotechnology}

moieties. Bio-orthogonal strategies would enable the multistep biochemical modification of engineered cellular microniches, which unlocks the single-cell-laden microgel as a versatile multipurpose platform for engineering cellular microenvironments. Furthermore, cytocompatible on-demand inducible and reversible crosslinking (or even pulsatile) strategies could endow microgels with controlled dynamic biophysical properties, such as in situ softening [28], stiffening [29], or stress relaxation [30], which can accurately control (stem) cell behavior.

Besides mimicking the extracellular matrix, hydrogels can also be endowed with functional moieties to emulate cell-cell interactions. For example, Burdick and coworkers demonstrated that stem cell behavior can be influenced by endowing a hydrogel with cadherin peptides that mimicked cell-cell adhesion, thereby improving the early chondrogenesis of mesenchymal stem cells [31]. We foresee that further modifying the single-cell microgel to mimic cell-cell interactions will be key to eventually emulating the cellular microenvironment of many native tissues.

\section{Fabrication Strategies to Generate Single-Cell Microgels}

The production of single-cell-laden microgels typically involves two critical steps: (i) the dispersion of the cell-laden hydrogel precursor solution into discrete droplets; and (ii) the gelation of the droplets through in situ crosslinking. Hydrogel precursor droplets can be formed via patterning or molding on and/or in solid substrates, emulsification in an (immiscible) liquid, or atomization in a gas $[32,33]$. Emulsification and atomization are continuous processes with inherently high throughputs, albeit with limited shape control; complex morphologies are difficult to produce, because a spherical morphology is energetically favored due to the surface tension. In response, semicontinuous stop flow lithography (SFL) approaches based on rapid in situ photocrosslinking with alternating 'stop-motion' flow have been developed to create microgels with almost any morphology [34]. However, there is as of yet no clear indication that nonspherical single-cell-laden microgels have advantages for biomedical applications (Box 2). Therefore, emulsification and atomization are currently considered as the most potent technologies for (large volume) clinical and industrial applications.

Opportunely, emulsified hydrogel precursor droplet can be stabilized with surfactants, so that a range of relatively slow (seconds to minutes) gelation mechanisms can be used including photocrosslinking [11], thermal gelation [35], enzymatic crosslinking [36], and Michael-type addition [37] reactions. However, despite the use of surfactants, cell-laden emulsions are prone to rapid destabilization due to the adsorption of biomolecules and cells onto the water-oil interface of the droplets, which limits the time needed for most in situ crosslinking mechanisms. Various strategies have been developed to stabilize the water-oil interface of (single-)cell-laden hydrogel precursor droplets, thereby preventing their de-emulsification and consequently extending the window of time for in situ crosslinking. For example, PEGylated surfactants can prevent the adsorption of biomolecules [38]. Furthermore, a dual photoinitiator strategy that presents crosslinking initiators in both the water and oil phases can rapidly crosslink the surface of the hydrogel precursor droplet from both the inside and the outside [11]. However, cells, thereby providing isotropic 3D biochemical and biophysical stimuli; (ii) result in less clogging compared with irregularly shaped microparticles, which, for example, enables injection of higher microgel concentrations using thinner needles [87]; (iii) cause less intense inflammatory responses upon in vivo implantation [88]; and (iv) intrinsically form predictable and well-controlled interconnected networks upon stacking, which promotes solute diffusion and tissue ingrowth (e.g., vascularization) [85]. 


\section{Trends in Biotechnology}

oils and surfactants affect cell behavior on the long term. Therefore, almost any cell-based application requires de-emulsification and extensive washing steps once the cells have been stably embedded in the hydrogel matrix. Various de-emulsification strategies for (single-) cellladen microgels have recently been developed, including on-chip electrocoalescence [39], offchip de-emulsification on hydrophobic paper [40], and spontaneous de-emulsification using ultrathin double emulsions [41].

In contrast to emulsification, atomization does not require the use of an immiscible liquid (e.g., oil) and surfactants. However, the lack of droplet stabilization limits this approach to the use of extremely rapid (milliseconds) crosslinking mechanisms. In practice, therefore, atomization is almost exclusively combined with ionic crosslinking strategies, such as alginate with divalent cations. Yet, hydrogels that rely on slower (seconds to minutes) crosslinking mechanisms can still be utilized by leveraging alginate as a sacrificial structural template in the form of an alginate shell or interpenetrating alginate network $[42,43]$.

Hydrogel crosslinking can be categorized based on the molecular interactions between the polymers, which are of a chemical or physical nature. Chemical interactions are formed by (chemically) reacting moieties yielding a permanent covalent bond. Traditionally, hydrogels are formed via the radical polymerization of monomers [44]. Radical-based crosslinking is often associated with cytotoxicity, unless radicals are efficiently consumed through enzymatic crosslinking, which is used, for example, in peroxidase-mediated crosslinking of phenolic moieties [45]. Expediently, radical-based single-cell encapsulation has also been achieved in a cytocompatible manner by adding a radical-forming photoinitiator to the immiscible oil phase rather than to the cell-laden hydrogel precursor phase. This strategy enabled microgel crosslinking in an outside-in manner, thereby minimizing the exposure of the encapsulated cell to cytotoxic radicals [11]. Physical bonds are reversible (i.e., noncovalent) and are, among others, based on entanglements, electrostatic (i.e., ionic or hydrogen bonds), van der Waals, or hydrophobic interactions, or a combination thereof. In particular, ionic crosslinking of alginate using divalent ions has been widely used for the generation of single-cell-laden microgels [46-51]. Crosby and coworkers leveraged the reversible nature of physical interactions for the controlled release and facile analysis of single-cell components by dissolving barium-crosslinked alginate microgels using sulfate as a barium chelator [52]. Reversible physical crosslinking can also endow hydrogels with self-healing properties that are, for example, key to provide a hydrogel with stress relaxation properties, which have recently been proven essential to trigger physiological cell responses in cell-laden bulk constructs [30]. Furthermore, reversible bonds enable the formulation of smart (i.e., stimulus-responsive) hydrogels, which are key to emulate the dynamic nature of the native pericellular matrix [53]. Indeed, several strategies based on both dynamic physical and chemical interactions have recently been proposed for the spatiotemporal functionalization of bulk hydrogels $[25,54]$. We anticipate that these smart materials will also soon be explored for 3D single-cell experimentation with spatiotemporal control over the cellular microenvironment (see also the 'Composition of the Single-Cell Microgel' section).

\section{Balancing Throughput and Size}

An important selection criterion for the fabrication method of cell-laden microgels is to a compromise between throughput and size. Here, we provide a quantitative technological comparison of the most used continuous droplet manufacturing techniques for cell-laden microgel fabrication: droplet microfluidics, vibrating jet, inkjet, jet cutting, electrospraying, and air-induced spraying (Figure 3 ). 


\section{Trends in Biotechnology}
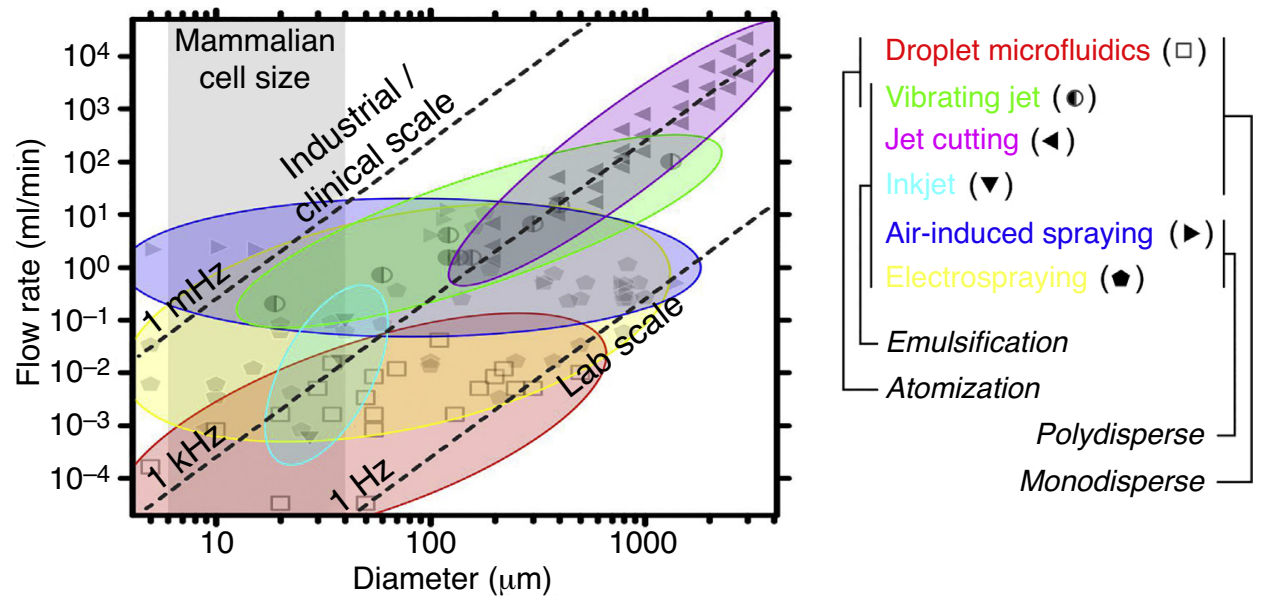

Trends in Biotechnology

Figure 3. Quantitative Landscape of Microdroplet Production Rate versus Size Range. A comprehensive overview of the typical per-nozzle throughput (i.e., flow rate) as a function of the droplet size (i.e., diameter) of the most used continuous droplet manufacturing techniques for cell-laden microgel fabrication is presented. The indicated production regimes are based on data points obtained from the following references: droplet microfluidics [60,91-98], vibrating jet [43], jet cutting [99,100], inkjet [101-103], air-induced spraying [100,104-107], and electrospraying [100,108-110]. If possible, multiple distinct data points were obtained from the mentioned studies. Furthermore, the mechanism of droplet formation (emulsification or atomization) and the monodisperse or polydisperse character of each fabrication method are indicated.

Spray-based encapsulation technologies (i.e., electro- and air-induced spraying) are ideal for both low- and high-throughput manufacturing of cell-sized microgels, but they typically produce polydisperse particles due to random liquid jet breakup. Notably, monodisperse microgels are preferred for most applications because they have superior defined pharmacokinetic properties in terms of both biodistribution and drug release (e.g., lower burst release) compared with polydisperse microgels [55]. Jet cutting produces monodisperse particles, but is not compatible with the manufacturing of sub-100- $\mu \mathrm{m}$ microgels, which is the most favorable size range for single-cell applications (see the 'Size Matters: Advantages of Hydrogel Miniaturization' section and Figure 1). By contrast, droplet microfluidics, vibrating jet, and inkjet technologies are perfectly suited for the production of monodispere single-cell-laden microgels smaller than $100 \mu \mathrm{m}$. Importantly, conventional chip-based microfluidic generation of droplets with diameters between 10 and $100 \mu \mathrm{m}$ yields a typical limited per-nozzle throughput of 1$10 \mu \mathrm{l} / \mathrm{min}$ (for the dispersed phase), because monodisperse emulsification is restricted to the dripping regimen [56]. A recent study transcended this limit by more than tenfold by using bubble-triggered breakup of cell-laden hydrogel precursor solution jets within a microfluidic chip [57]. However, the presence of air bubbles within microfluidic chips is also associated with significant flow instabilities [58]. Alternative rapid jet-based microfabrication methods that do not rely on microfluidic chips have also been explored, including inkjet [46] and vibrating jet technologies [43]. Altogether, we anticipate that rapid monodisperse jet-based microgel fabrication technologies will drive the clinical and industrial translation of single-cell microgel technologies, while slower but equally accurate (chip-based) microfluidic approaches will continue to support lab-scale applications.

How to Obtain Pure Single-Cell Microgel Populations

Cell encapsulation in droplets is typically a stochastic process that follows Poisson statistics, which intrinsically limits the maximum single-cell encapsulation yield to 37\% (Figure 4A) [59]. 


\section{Trends in Biotechnology}
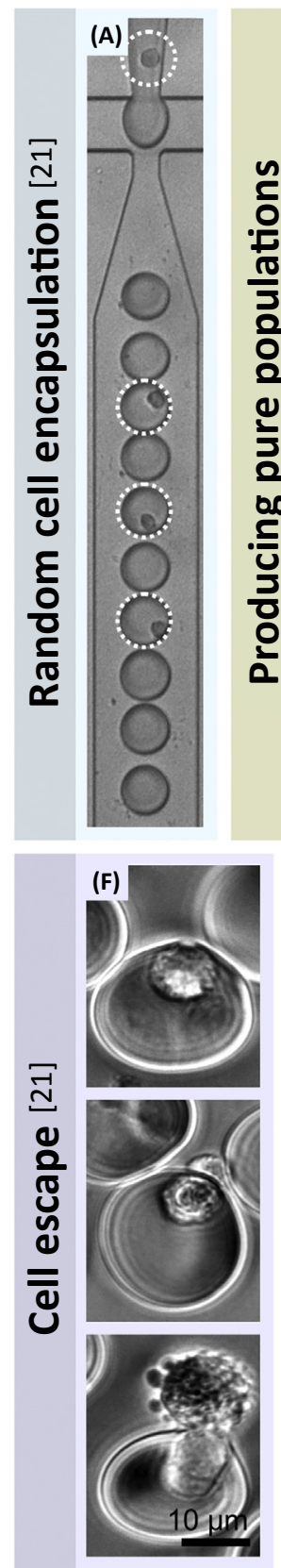

(B) Postproduction enrichment [11]

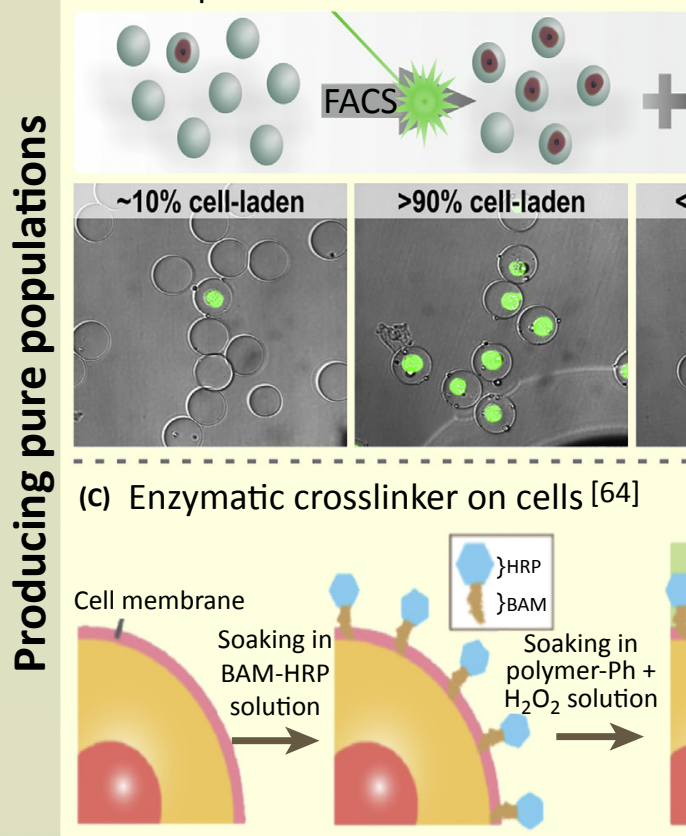

(G) Dual encapsulation [20]

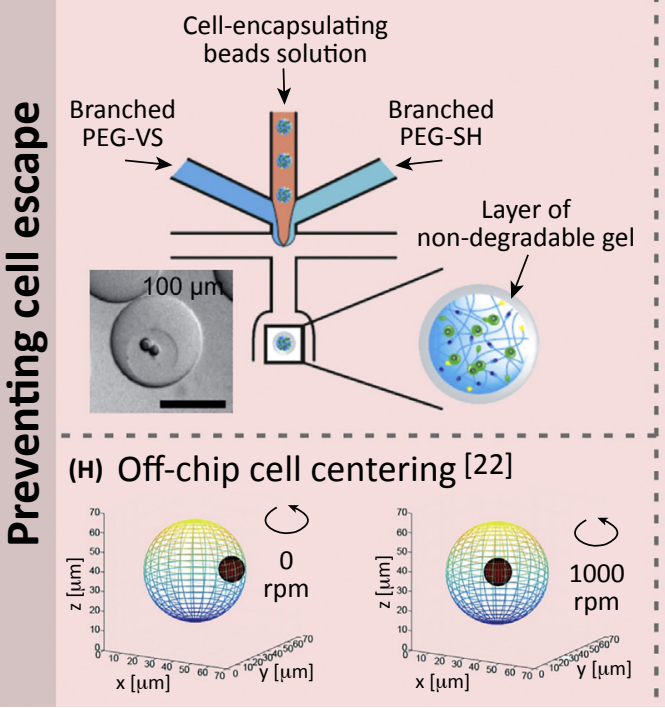

(D) Crosslinker precursor on cells [10]
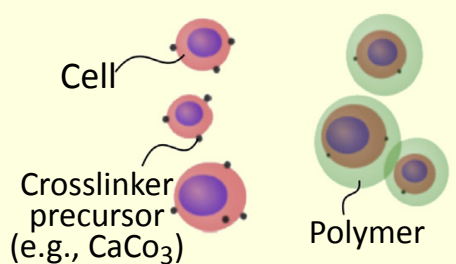

(E) Enzyme cofactor on cells [22]
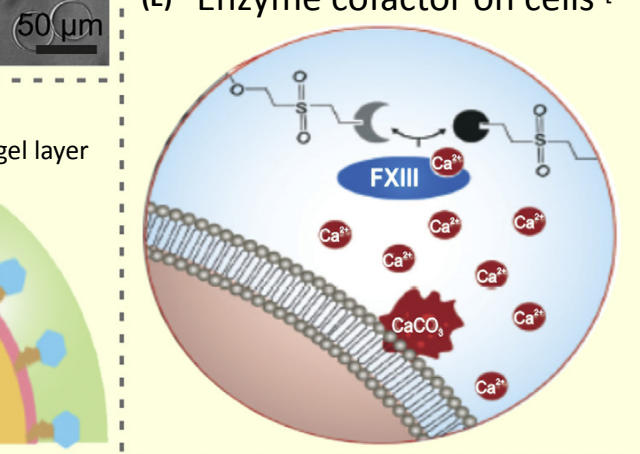

(I) On-chip cell centering [21]

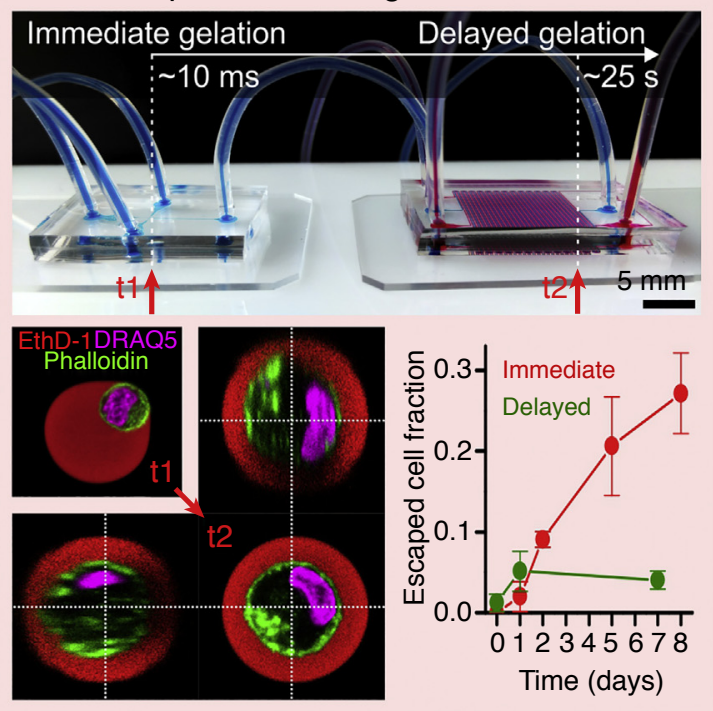

Trends in Biotechnology

Figure 4. Technological Advances to Realize Pure and Long-Term Single-Cell Microgel Cultures. (A) Random cell encapsulation [21] is described by Poisson statistics, which inherently results in maximally $37 \%$ single-cell-laden microgels and typically many empty (i.e., cell-free) microgels. (B) Fluorescence-activated cell sorting (FACS) can be used to enrich single-cell-laden microgel population by sorting out the empty microgels (postproduction enrichment [11]). Alternatively, pure single-cell microgel populations can be obtained via selective crosslinking cell-laden microgels, which is achieved by adsorbing onto the cytoplasmic membrane of the cell (C) an enzymatic crosslinker [64], (D) a crosslinker precursor [10], or (E) a cofactor of a crosslinking enzyme [22]. (F) Conventional microencapsulation strategies result in off-center cell encapsulation, which causes cell escape upon culture [21]. Cell escape can be prevented by (G) applying a nondegradable hydrogel coating [20], and by positioning the cells in the center of the microdroplets before crosslinking using $(\mathrm{H})$ off-chip mixing of the emulsion [22] or (I) delayed on-chip crosslinking of the cell-laden prepolymer droplets continuously flowing within a microfluidic channel [21]. Images adapted, with permission, from [10,11,20-22,64]. 


\section{Trends in Biotechnology}

Although high-yield deterministic single-cell encapsulation in aqueous droplets has been achieved using inertial focusing [60], the forces involved in this process are too weak to obtain longitudinal cell ordering in comparatively viscous fluids, such as hydrogel precursor solutions. Opportunely, several innovative strategies have recently been developed to overcome the limitations of random (i.e., Poisson-distributed) cell encapsulation. For example, some studies have demonstrated that postproduction sorting of single-cell-laden microgels using flow cytometry yields near-pure cell-laden microgel populations (Figure 4B) [10,11]. We anticipate that alternative active sorting methods that have already been successfully applied in combination with droplet microfluidics, such as dielectrophoresis [61], acoustophoresis [62], and deterministic lateral displacement [63], will soon be integrated with microgel technology for the postproduction sorting of single-cell-laden microgels. Alternatively, deterministic single-cell encapsulation has been demonstrated using exclusive crosslinking of cell-laden microdroplets. For example, Sakai and coworkers exploited cellular membrane anchoring of a peroxidase to mediate the enzymatic crosslinking of phenol-functionalized alginate droplets (Figure 4C) [64]. The groups of Weitz and Mooney similarly achieved selective ionic crosslinking of alginate microgels (Figure 4D) and selective enzymatic crosslinking of glutamine and lysine-functionalized PEG by transglutaminase using calcium carbonate nanoparticle cell coatings (Figure 4E) to exclusively crosslink cell-laden microdroplets $[10,22]$.

To encapsulate single cells in microgels, cell aggregation must be prevented. Gravity-induced sedimentation of cells is a key contributor to cell aggregation. Cell sedimentation can be prevented by matching the microgel precursor solution and cellular densities by supplementing the prepolymer with a density gradient medium, such as iodixanol, also known as OptiPrep $[2,20]$. However, the use of iodixanol should be avoided when using photocrosslinking-based strategies, because this combination results in the production of the cytotoxic compound iodine. Alternatively, photoinert colloidal silica particles, such as Percoll, can be considered [11]. Cell aggregation may also be induced by the DNA released from lysed cells, which can be effectively prevented by the use of DNA restriction enzymes, such as DNase 1 [2].

How to Prevent Cell Escape

Microgel technology has the potential to provide single cells with biochemical and biophysical stimuli in a 3D isotropic manner. However, traditional microencapsulation strategies have failed to provide this feature due to off-center cell encapsulation. Moreover, off-center encapsulation is associated with the rapid egression of cells from the microgels (Figure 4F) [10,21]. Cell egression from microgels can be controlled by adapting the molecular weight of the polymer [10], supplementing low concentrations of the hydrogel precursor (i.e., monomers) to the medium [19], or adding an extra layer of permanent (i.e., nondegradable) hydrogel (Figure 4G) $[20,65]$. Given that cell escape from microgels correlates with off-center cell encapsulation, it could also be prevented by encapsulating cells in the center of microgels. Cell centering in microgels can be achieved in an off-chip and an on-chip manner. Off-chip cell centering has been achieved by placing the crosslinking hydrogel droplets on an orbital shaker (Figure $4 \mathrm{H}$ ) [22]. On-chip cell centering has been demonstrated using a microfluidic delay line that enabled the delayed in situ crosslinking of flowing droplets, which effectively repositioned the cells towards the centers of the droplets before gelation was initiated (Figure 4I) [21]. Cell centering prevents cell egression, which enables the long-term 3D culture ( $\geq 28$ days) of single-cell-laden microgels smaller than $50 \mu \mathrm{m}$ [21].

\section{Applications of Single-Cell Microgel Technology}

Single-cell microgel technology is of particular interest for several life science applications. Cellular microniches with a user-defined composition show great promise, for example, to 


\section{Trends in Biotechnology}

(A) Controlling stem cell fate

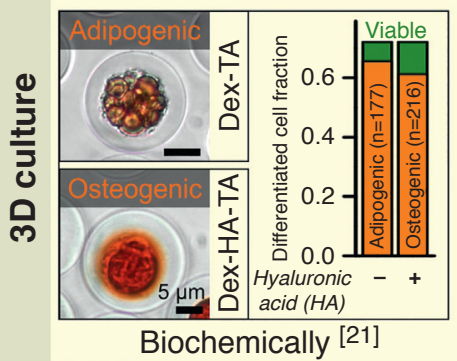

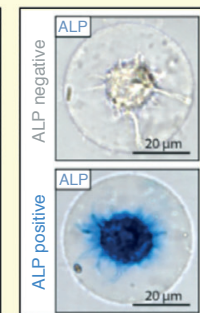

Biophysically [22]
(B) Screening microgel composition [66]

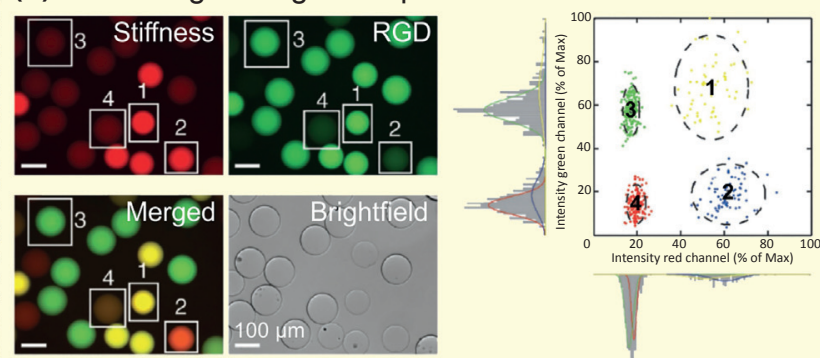

(C) In situ capturing cellular compounds [76]
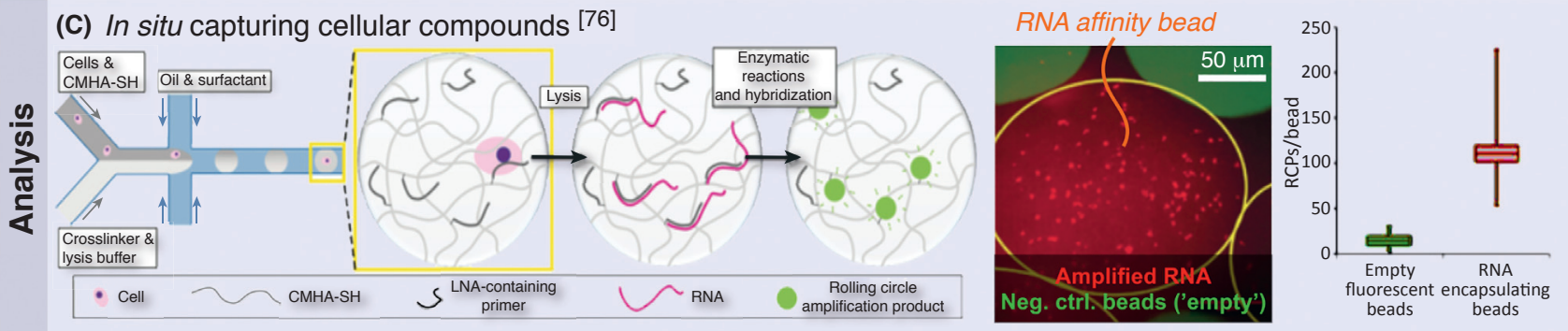

(D) Stem cell injections [10]

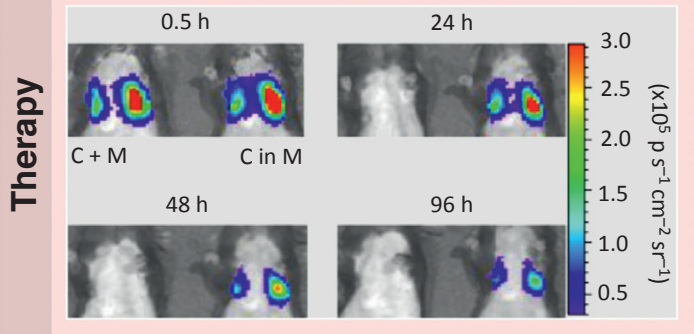

(E) Modular tissue engineering [11]
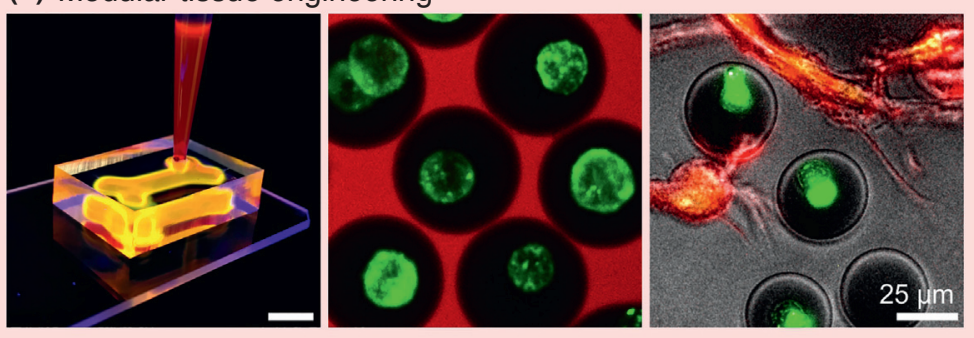

Trends in Biotechnology

Figure 5. Examples of Single-Cell Microgel Technology. (A) Single-cell 3D microgel platforms have been used to control the fate of stem cells by tuning the biochemical [21] and biophysical [22] properties of the microgels. (B) Microfluidic mixers have been used to generate compositional microgel libraries, where fluorescent labeling enabled the flow cytometry-based identification of specific biochemical and biophysical compositions post production [66]. (C) Specific cellular compounds, such as RNA, can be captured, amplified, and quantified in situ by endowing single-cell microgels with affinity molecules, thereby yielding so-called 'affinity' microbeads [76]. (D) The detectable in vivo retention time of intravenously injected stem cells is significantly increased by encapsulating the cells in microgels [10]. (E) A modular bioink was developed by incorporating single-cell microgels in an injectable hydrogel precursor solution [11]. Injection molding resulted in a modular tissue construct that was characterized by independently tunable micro- and macroenvironments. This single-cell modular tissue-engineering strategy was leveraged to incorporate immunoprotective single-cell-laden microgels within a proangiogenic macrogel that supported prevascular cellular network formation. Images adapted, with permission, from $[10,11,21,22,66,76]$.

study cell-material interactions in a physiologically relevant 3D microenvironment. The biochemical and biophysical composition of a microgel are instrumental in controlling cellular survival, behavior, and function. For example, they can be tuned to steer stem cell lineage commitment (Figure 5A) [18,21,22], as mentioned previously. Integrating single-cell microgel technology with advanced microfluidic platforms has enabled the efficient screening of multiple microniche compositions. For instance, Lutolf and coworkers developed a dedicated microfluidic device to mix and emulsify up to five different materials to produce a small library of (cellfree) microgels (Figure 5B) [66]. After fluorescent encoding of the distinct materials, the different microniches were successfully identified and selected in a high-throughput manner using flow cytometry. In a different approach, an array of approximately 1500 multicell-laden microgels 


\section{Trends in Biotechnology}

was created in a microfluidic device, each microgel being anchored in a 2-nL microwell. This elegant platform allowed for the tracking and time-lapse imaging of the cells in the individual microgels, as well as their in situ stimulation by perfusing chemicals in the main channel above the microwell array [67]. This platform was recently applied to establish an antibiogram by exposing monoclonal bacteria colonies (i.e., originating from single-cell microgels) to a gradient of antibiotics [68]. Subsequently, bacteria could be selectively retrieved from the platform by the on-demand melting of specific microgels for off-chip genetic analysis. Furthermore, this screening platform was utilized to optimize the transient transfection of single cells using a library of cationic lipoplexes [69].

Droplet microfluidics has been acknowledged as a powerful tool for the massive upscaling of single-cell 'omics analysis, as demonstrated by several droplet-based approaches, including 'Drop-seq' [70], 'inDrop' [71], and CytoSeq' [72]. However, the complexity of the analytical workflow is limited when using droplet microfluidics because this technology is not readily compatible with solution exchange, which is essential in various 'omics approaches. Furthermore, the long-term study of adherent cells is precluded in an all-liquid environment. These limitations can be avoided by the use of 3D hydrogel matrices instead of aqueous solutions. For example, Mathies and coworkers captured the genetic material from single cells by coencapsulating cells with primer-conjugated microbeads in agarose microgels [73]. By combining various emulsification, in situ gelation, and de-emulsification steps, they achieved the successive encapsulation, lysis, gene amplification, and sequencing of individual cells. Using this method, genetically altered cells could be accurately identified within a heterogeneous cancer cell population. However, the co-encapsulation of cells and affinity beads is governed by double Poisson statistics, which considerably limits the screening efficiency because the number of microgels that contain both a single cell and a microbead is maximally $13.5 \%$. To increase the efficiency of the assay, Yang and coworkers directly immobilized cell-specific genetic capture sequences and primers on the agarose polymer, thereby enabling Poissondistributed single-cell encapsulation in affinity microgels (i.e., maximally $37 \%$ single-cell yield). After cell lysis, polymerase chain reactions (PCR) were performed in the liquid state (i.e., ungelled agarose), which further improved the single-cell genome analysis technology by increasing the PCR efficiency from $40 \%$ to $95 \%$. [74]. Subsequent gelation of the agarose ensured physical entrapment of the amplified DNA, which enabled the flow cytometry-based detection of rare pathogenic Escherichia coli within a population of nonpathogenic bacteria with high accuracy (1 in 100 000). This strategy was next extended to single-cell reverse transcription PCR, which enabled the quantification of the transcription levels of a cancer biomarker (i.e., EpCAM) in various cancer cell lines [75]. In an alternative approach to analyze single-cell gene expression while omitting the preamplification bias, Huck and coworkers implemented RNA rolling circle amplification within primer-conjugated microgels (Figure 5C) [76]. This strategy enabled facile RNA quantification by counting the number of fluorescently labeled rolling circle amplification products (I.e., 'RNA colonies') in the microgel. More recently, single-cell-laden microgels were combined with next-generation sequencing for single-cell whole-genome analysis [52]. Following similar affinity bead strategies, single-cell microdroplet and microgel technologies were used to isolate and/or detect specific compounds secreted by individual cells, including antibodies [2], lipids [77], cytokines [78], and proteases [79]. Altogether, the integration of such 'omics analysis strategies with single-cell microgels is driving the in-depth high-throughput probing of cell behavior in biomimetic controllable 3D microenvironments.

Other key fields in which single-cell microgel technology is likely to have a positive role in the near future are tissue engineering and regenerative medicine. Applications of special interest include (stem) cell injections, immunoprotection, and biofabrication. Although stem cells have 


\section{Trends in Biotechnology}

\section{Key Figure}

\section{Key Applications of Single-Cell-Laden Microgels}

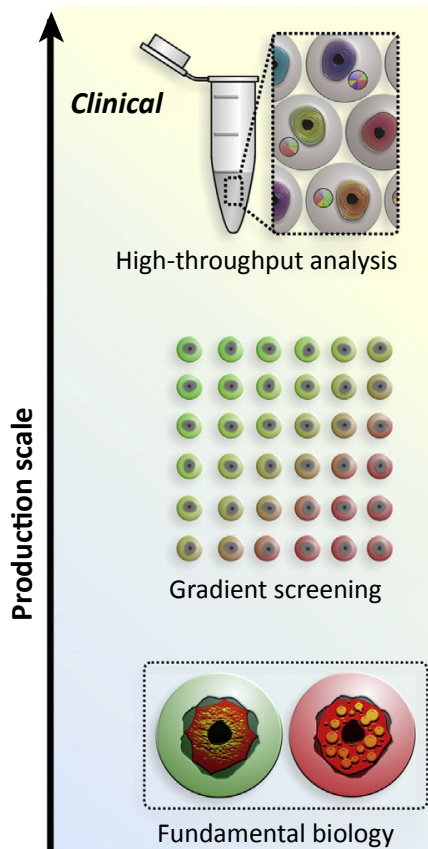

$L a b$

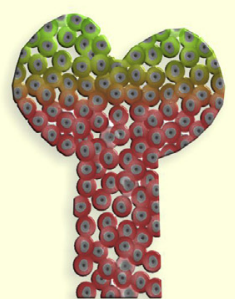

Bottom-up tissue engineering

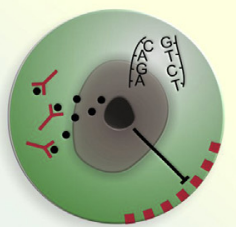

Cellular compound capturing

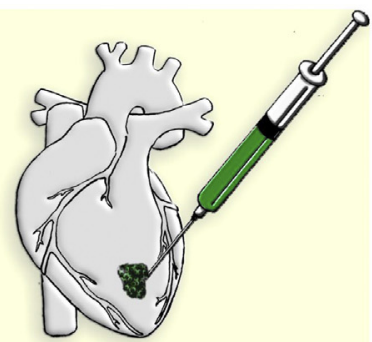

Cell injection therapies

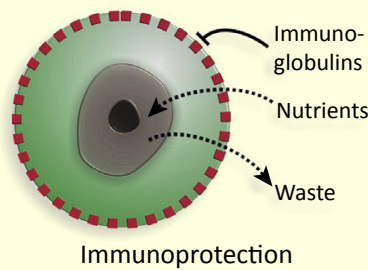

Therapeutic

Application area

Trends in Biotechnology

Figure 6. The application area (analytical versus therapeutic) and production scale (lab versus clinical) of several single-cell microgel applications are indicated. The applications include fundamental biology (e.g., for stem cell differentiation), gradient screening (e.g., via microgel anchoring), high-throughput analysis (e.g., using primer-based barcoding), cellular compound capturing (e.g., using DNA affinity beads), bottom-up tissue engineering (e.g., using single-cell building blocks), immunoprotection (e.g., for cell transplantation), and cell injection (e.g., for stem cell therapies).

significant potential as anti-inflammatory and trophic mediators [80], their therapeutic effect upon injection is hampered by fast cell clearance from the injection site and poor long-term cell survival $[81,82]$. Cell-laden microcarriers are characterized by improved retention after intramyocardial injection compared with bare cell suspensions [83]. Based on similar observations regarding the retention time (Figure 5D), and even a therapeutic advantage of single-cell-laden microgels over nonencapsulated cells and multicell-laden microgels [10], we postulate that single-cell microgel technology could further improve stem cell injection therapies by prolonging retention time, as well as improving cell survival by providing a protective microenvironment. Furthermore, the semipermeable nature of hydrogels could be used to provide cells with an immunoprotective microcoating [11]. Lastly, single-cell-laden microgels are of interest as building blocks for modular or bottom-up tissue-engineering strategies (Figure 5E). For example, microgels could be mixed with a distinct injectable hydrogel to form a modular bioink for 


\section{Trends in Biotechnology}

CellPress REVIEWS the facile fabrication of multiscale modular biomaterials with cell-specific microenvironments within a host-specific macroenvironment [11], which conceptually mimics the pericellular and extracellular matrices of native tissues. One of the key benefits of microgel-based tissue constructs is their inherent capacity to form interconnected networks. The resulting porous scaffold not only facilitates diffusion of nutrients and waste products [84], but also the formation of cellular networks, which can significantly accelerate wound healing compared with nonporous tissue constructs [85].

\section{Concluding Remarks}

Single-cell microgel technology enables the encapsulation of an individual cell in a hydrogel that is only a few micrometers larger than the cell itself. Their small size provides single-cell-laden microgels with several pharmacological and physiological advantages. Recent discoveries have enabled the long-term culture of living single-cell-laden microgels. Future research is expected to focus on biochemically and biophysically smart modifications of these microgels, which would aid the improved mimicry of the native pericellular matrix and more control over (stem) cell behavior within in vitro and in vivo single-cell applications (see Outstanding Questions). Furthermore, we anticipate that microgels will be endowed with moieties to mimic cell-cell interactions, which may eventually enable in vitro experimentation of single cells within a controlled microenvironment that emulates all biological, biochemical, and biophysical cues of the native cellular microenvironment. It is also expected that confining a cell within a cell-sized microniche will open new avenues for single-cell 'omics, because this approach facilitates the detection of low-abundant markers by limiting the dilution factor of the cellular contents. A major hurdle to translate single-cell microgel technologies towards clinical and industrial applications, is their limited production throughput. Rapid jet-based microencapsulation strategies are anticipated to drive the clinical and industrial translation of single-cell microgel technologies. As summarized in Figure 6 (Key Figure), single-cell microgel technology offers several advanced tools for culture and high-throughput analysis of single cells within biomimetic 3D microenvironments, as well as improved tissue engineering and cell-based therapies.

\section{Acknowledgments}

We thank Claas Willem Visser for his help with the quantitative technological comparison as presented in Figure 3. We thank Sieger Henke for carefully reading the manuscript. T.K., M.K., and J.L. acknowledge financial support from the MIRA Institute for Biomedical Technology and Technical Medicine. M.K. acknowledges financial support from the Dutch Arthritis Foundation (LLP-25). J.L. acknowledges financial support from an Innovative Research Incentives Scheme Veni award (\#14328) from the Netherlands Organization for Scientific Research (NWO), the European Research Council (ERC, Starting Grant, \#759425), and the Dutch Arthritis Foundation (\#17-1-405).

\section{References}

1. Rakszewska, A. et al. (2014) One drop at a time: toward droplet microfluidics as a versatile tool for single-cell analysis. Npg Asia Mater. 6, e133

2. Mazutis, L. et al. (2013) Single-cell analysis and sorting using droplet-based microfluidics. Nat. Protoc. 8, 870-891

3. Tibbitt, M.W. and Anseth, K.S. (2009) Hydrogels as extracellular matrix mimics for 3D cell culture. Biotechnol. Bioeng. 103, 655663

4. Ling, Y. et al. (2007) A cell-laden microfluidic hydrogel. Lab Chip $7,756-762$

5. Robitaille, R. et al. (1999) Studies on small (<350 microm) alginate-poly-L-lysine microcapsules. III. Biocompatibility of smaller versus standard microcapsules. J. Biomed. Mater. Res. 44, 116-120
6. Sakai, S. et al. (2006) Biocompatibility of subsieve-size capsules versus conventional-size microcapsules. J. Biomed. Mater. Res. A 78, 394-398 optical imaging frontier in biology. Nat. Methods 7, 603-614

8. Chicheportiche, D. and Reach, G. (1988) In vitro kinetics of insulin release by microencapsulated rat islets: effect of the size of the microcapsules. Diabetologia 31, 54-57

9. Headen, D.M. et al. (2014) Microfluidic-based generation of size-controlled, biofunctionalized synthetic polymer microgels for cell encapsulation. Adv. Mater. 26, 3003-3008

10. Mao, A.S. et al. (2017) Deterministic encapsulation of single cells in thin tunable microgels for niche modelling and therapeutic delivery. Nat. Mater. 16, 236-243
7. Ntziachristos, V. (2010) Going deeper than microscopy: the

\section{Outstanding Questions}

How can the cellular microenvironment be emulated in single-cell microgels in terms of spatiotemporal dynamics, interactive responsiveness, and cellcell contact?

Can cell egression from microgels be tuned and timed to control the in vivo delivery of cells?

For which applications are single-cellladen microgels more potent than larger multicell-laden hydrogel constructs?

How can the production of industrial or clinical quantities of single-cell-laden microgels be reached while maintaining high cell survival and functionality? 


\section{Trends in Biotechnology}

11. Kamperman, T. et al. (2017) Single cell microgel based modular bioinks for uncoupled cellular micro- and macroenvironments. Adv. Healthc. Mater. 6, 1600913

12. Del Monte, U. (2009) Does the cell number 10(9) still really fit one gram of tumor tissue? Cell Cycle 8, 505-506

13. Quinn, T.M. et al. (2013) Cell and matrix morphology in articular cartilage from adult human knee and ankle joints suggests depth-associated adaptations to biomechanical and anatomical roles. Osteoarthritis Cartilage 21, 1904-1912

14. Miller, C.C. et al. (2003) Validation of a morphometric method for evaluating fibroblast numbers in normal and pathologic tissues. Exp. Dermatol. 12, 403-411

15. Vajanto, I. et al. (2002) Evaluation of angiogenesis and side effects in ischemic rabbit hindlimbs after intramuscular injection of adenoviral vectors encoding VEGF and LacZ. J. Gene Med. 4, 371-380

16. Carragee, E.J. et al. (2011) A critical review of recombinant human bone morphogenetic protein-2 trials in spinal surgery: emerging safety concerns and lessons learned. Spine J. 11, $471-491$

17. Ma, S. et al. (2013) Monodisperse collagen-gelatin beads as potential platforms for 3D cell culturing. J. Mater. Chem. B 1 , 5128

18. Ma, Y. et al. (2014) Artificial microniches for probing mesenchymal stem cell fate in 3D. Biomater. Sci. 2, 1661-1671

19. Dolega, M.E. et al. (2015) Controlled 3D culture in Matrigel microbeads to analyze clonal acinar development. Biomaterials $52,347-357$

20. Allazetta, S. et al. (2015) Cell-instructive microgels with tailormade physicochemical properties. Small 11, 5647-5656

21. Kamperman, T. et al. (2017) Centering single cells in microgels via delayed crosslinking supports long-term 3D culture by preventing cell escape. Small 13, 1603711

22. Lienemann, P.S. et al. (2017) Single cell-laden protease-sensitive microniches for long-term culture in 3D. Lab Chip 17, 727-737

23. Khetan, S. et al. (2013) Degradation-mediated cellular traction directs stem cell fate in covalently crosslinked three-dimensional hydrogels. Nat. Mater. 12, 458-465

24. Buwalda, S.J. et al. (2014) Hydrogels in a historical perspective: from simple networks to smart materials. J. Control. Release 190, 254-273

25. Brown, T.E. and Anseth, K.S. (2017) Spatiotemporal hydrogel biomaterials for regenerative medicine. Chem. Soc. Rev. 46, 6532-6552

26. Rosales, A.M. and Anseth, K.S. (2016) The design of reversible hydrogels to capture extracellular matrix dynamics. Nat. Rev. Mater. 1, 15012

27. Burdick, J.A. and Murphy, W.L. (2012) Moving from static to dynamic complexity in hydrogel design. Nat. Commun. 3, 1269

28. Yang, C. et al. (2014) Mechanical memory and dosing influence stem cell fate. Nat. Mater. 13, 645-652

29. Guvendiren, M. and Burdick, J.A. (2012) Stiffening hydrogels to probe short- and long-term cellular responses to dynamic mechanics. Nat. Commun. 3, 792

30. Chaudhuri, O. et al. (2016) Hydrogels with tunable stress relaxation regulate stem cell fate and activity. Nat. Mater. 15, 326334

31. Bian, L. et al. (2013) Hydrogels that mimic developmentally relevant matrix and $\mathrm{N}$-cadherin interactions enhance MSC chondrogenesis. Proc. Natl. Acad. Sci. U. S. A. 110, 10117-10122

32. Tran, V.-T. et al. (2011) Why and how to prepare biodegradable, monodispersed, polymeric microparticles in the field of pharmacy? Int. J. Pharm. 407, 1-11

33. Lima, A.C. et al. (2012) Production methodologies of polymeric and hydrogel particles for drug delivery applications. Expert Opin. Drug Deliv. 9, 231-248
34. Panda, P. et al. (2008) Stop-flow lithography to generate cellladen microgel particles. Lab Chip 8, 1056-1061

35. Kumachev, A. et al. (2011) High-throughput generation of hydrogel microbeads with varying elasticity for cell encapsulation. Biomaterials 32, 1477-1483

36. Kamperman, T. et al. (2017) Nanoemulsion-induced enzymatic crosslinking of tyramine-functionalized polymer droplets. J. Mater. Chem. B 5, 4835-4844

37. Rossow, T. et al. (2012) Controlled synthesis of cell-laden microgels by radical-free gelation in droplet microfluidics. J. Am. Chem. Soc. 134, 4983-4989

38. Holtze, C. et al. (2008) Biocompatible surfactants for water-influorocarbon emulsions. Lab Chip 8, 1632-1639

39. Chokkalingam, V. et al. (2014) An electro-coalescence chip fo effective emulsion breaking in droplet microfluidics. Lab Chip 14, 2398-2402

40. Lee, D.H. et al. (2014) Rapid one-step purification of single-cells encapsulated in alginate microcapsules from oil to aqueous phase using a hydrophobic filter paper: implications for single-cell experiments. Biotechnol. J. 9, 1233-1240

41. Choi, C.H. et al. (2016) One-step generation of cell-laden microgels using double emulsion drops with a sacrificial ultra-thin of shell. Lab Chip 16, 1549-1555

42. Tamayol, A. et al. (2015) Hydrogel templates for rapid manufacturing of bioactive fibers and 3D constructs. Adv Healthc. Mater. 4, 2146-2153

43. Visser, C.W. et al. (2018) In-air microfluidics enables rapid fabrication of emulsions, suspensions, and 3D modular (bio)materials. Sci. Adv. 4, eaao1175

44. Wichterle, O. and LíM, D. (1960) Hydrophilic gels for biologica use. Nature 185, 117-118

45. Henke, S. et al. (2016) Enzymatic crosslinking of polymer conjugates is superior over lonic or UV crosslinking for the on-chip production of cell-laden microgels. Macromol. Biosci. 16, 15241532

46. $\mathrm{Xu}, \mathrm{T}$. et al. (2008) High-throughput production of single-cel microparticles using an inkjet printing technology. J. Manuf. Sci. Eng. 130, 021017

47. Liu, K etal (2012) Generation of disk-like hydrogel beads for cell encapsulation and manipulation using a droplet-based microfluidic device. Microfluid. Nanofluid. 13, 761-767

48. Martinez, C.J. et al. (2012) A microfluidic approach to encapsulate living cells in uniform alginate hydrogel microparticles. Mac romol. Biosci. 12, 946-951

49. Akbari, S. and Pirbodaghi, T. (2013) Microfluidic encapsulation of cells in alginate particles via an improved internal gelation approach. Microfluid. Nanofluid. 16, 773-777

50. Utech, S. et al. (2015) Microfluidic generation of monodisperse, structurally homogeneous alginate microgels for cell encapsulation and 3D cell culture. Adv. Healthc. Mater. 4, 1628-1633

51. Onoe, H. et al. (2014) Centrifuge-based cell encapsulation in hydrogel microbeads using sub-microliter sample solution. RSC Adv. 4, 30480-30484

52. Bigdeli, S. et al. (2015) A simple method for encapsulating single cells in alginate microspheres allows for direct PCR and whole genome amplification. PLoS One 10, e0117738

53. Vats, K. and Benoit, D.S. (2013) Dynamic manipulation of hydrogels to control cell behavior: a review. Tissue Eng. Part B Rev. 19, 455-469

54. Uto, K. et al. (2017) Dynamically tunable cell culture platforms for tissue engineering and mechanobiology. Prog. Polym. Sci. 65 $53-82$

55. Xu, Q. et al. (2009) Preparation of monodisperse biodegradable polymer microparticles using a microfluidic flow-focusing device for controlled drug delivery. Small 5, 1575-1581

56. Nunes, J.K. et al. (2013) Dripping and jetting in microfluidic multiphase flows applied to particle and fiber synthesis. J. Phys. D Appl. Phys. 46, 114002 


\section{Trends in Biotechnology}

57. Yan, Z. et al. (2017) Rapid encapsulation of cell and polymer solutions with bubble-triggered droplet generation. Macromol. Chem. Phys. 218, 1600297

58. van Steijn, V. et al. (2008) Velocity fluctuations of segmented flow in microchannels. Chem. Eng. J. 135, S159-S165

59. Collins, D.J. et al. (2015) The Poisson distribution and beyond: methods for microfluidic droplet production and single cell encapsulation. Lab Chip 15, 3439-3459

60. Kemna, E.W. et al. (2012) High-yield cell ordering and deterministic cell-in-droplet encapsulation using Dean flow in a curved microchannel. Lab Chip 12, 2881-2887

61. Baret, J.C. et al. (2009) Fluorescence-activated droplet sorting (FADS): efficient microfluidic cell sorting based on enzymatic activity. Lab Chip 9, 1850-1858

62. Schmid, L. et al. (2014) Sorting drops and cells with acoustics: acoustic microfluidic fluorescence-activated cell sorter. Lab Chip 14, 3710-3718

63. Jing, T. et al. (2015) Jetting microfluidics with size-sorting capability for single-cell protease detection. Biosens. Bioelectron. 66, 19-23

64. Sakai, S. and Taya, M. (2014) On-cell surface cross-linking of polymer molecules by horseradish peroxidase anchored to cell membrane for individual cell encapsulation in hydrogel sheath. ACS Macro Lett. 3, 972-975

65. Ma, M. et al. (2013) Core-shell hydrogel microcapsules for improved islets encapsulation. Adv. Healthc. Mater. 2, 667-672

66. Allazetta, S. et al. (2017) Microfluidic programming of compositional hydrogel landscapes. Macromol. Rapid Commun. 38, 1700255

67. Sart, S. et al. (2017) Multiscale cytometry and regulation of 3D cell cultures on a chip. Nat. Commun. 8, 469

68. Amselem, G. et al. (2016) Universal microfluidic platform for bioassays in anchored droplets. Lab Chip 16, 4200-4211

69. Vitor, M.T. et al. (2018) Tracking the evolution of transiently transfected individual cells in a microfluidic platform. Sci. Rep. 8,1225

70. Macosko, E.Z. et al. (2015) Highly parallel genome-wide expression profiling of individual cells using nanoliter droplets. Cell 161 , 1202-1214

71. Klein, A.M. et al. (2015) Droplet barcoding for single-cell transcriptomics applied to embryonic stem cells. Cell 161, 11871201

72. Fan, H.C. et al. (2015) Expression profiling. Combinatorial labeling of single cells for gene expression cytometry. Science 347 1258367

73. Novak, R. et al. (2011) Single-cell multiplex gene detection and sequencing with microfluidically generated agarose emulsions. Angew. Chem. Int. Ed. Engl. 50, 390-395

74. Zhu, Z. et al. (2012) Highly sensitive and quantitative detection of rare pathogens through agarose droplet microfluidic emulsion PCR at the single-cell level. Lab Chip 12, 3907-3913

75. Zhang, H. et al. (2012) Massively parallel single-molecule and single-cell emulsion reverse transcription polymerase chain reaction using agarose droplet microfluidics. Anal. Chem. 84 , 3599-3606

76. Rakszewska, A. et al. (2016) Quantitative single-cell mRNA analysis in hydrogel beads. Angew. Chem. Int. Ed. Engl. 55, 6698-6701

77. Lee, D.H. et al. (2013) In situ analysis of heterogeneity in the lipid content of single green microalgae in alginate hydrogel microcapsules. Anal. Chem. 85, 8749-8756

78. Chokkalingam, V. et al. (2013) Probing cellular heterogeneity in cytokine-secreting immune cells using droplet-based microfluidics. Lab Chip 13, 4740-4744

79. Son, K.J. et al. (2015) A microsystem integrating photodegradable hydrogel microstructures and reconfigurable microfluidics for single-cell analysis and retrieval. Lab Chip 15, 637-641

80. Caplan, A.I. and Dennis, J.E. (2006) Mesenchymal stem cells as trophic mediators. J. Cell Biochem. 98, 1076-1084
81. Feyen, D.A. et al. (2016) Stem cell-based therapy: improving myocardial cell delivery. Adv. Drug Deliv. Rev. 106, 104-115

82. Ansboro, S. et al. (2012) Strategies for improved targeting of therapeutic cells: implications for tissue repair. Eur. Cell Mater. 23, 310-318, discussion 318-319

83. van den Akker, F. et al. (2017) Intramyocardial stem cell injection: go(ne) with the flow. Eur. Heart J. 38, 184-186

84. Matsunaga, Y.T. et al. (2011) Molding cell beads for rapid construction of macroscopic 3D tissue architecture. Adv. Mater. 23, $\mathrm{H} 90-\mathrm{H} 94$

85. Griffin, D.R. et al. (2015) Accelerated wound healing by injectable microporous gel scaffolds assembled from annealed building blocks. Nat. Mater. 14, 737-744

86. Baker, B.M. and Chen, C.S. (2012) Deconstructing the third dimension: how 3D culture microenvironments alter cellular cues. J. Cell Sci. 125, 3015-3024

87. Caine, M. et al. (2017) Review of the development of methods for characterization of microspheres for use in embolotherapy: translating bench to cathlab. Adv. Healthc. Mater. 6, 1601291

88. Veiseh, O. et al. (2015) Size- and shape-dependent foreign body immune response to materials implanted in rodents and nonhuman primates. Nat. Mater. 14, 643-651

89. Pradal, J. et al. (2016) Effect of particle size on the biodistribution of nano- and microparticles following intra-articular injection in mice. Int. J. Pharm. 498, 119-129

90. Formiga, F.R. et al. (2013) Biodegradation and heart retention of polymeric microparticles in a rat model of myocardial ischemia. Eur. J. Pharm. Biopharm. 85, 665-672

91. Dendukuri, D. and Doyle, P.S. (2009) The synthesis and assembly of polymeric microparticles using microfluidics. Adv. Mater. 21, 4071-4086

92. Femmer, T. et al. (2015) High-throughput generation of emul sions and microgels in parallelized microfluidic drop-makers prepared by rapid prototyping. ACS Appl. Mater. Interfaces 7. 12635-12638

93. Tumarkin, E. et al. (2011) High-throughput combinatorial cell coculture using microfluidics. Integr. Biol. 3, 653-662

94. Yobas, L. et al. (2006) High-performance flow-focusing geometry for spontaneous generation of monodispersed droplets. Lab Chip 6, 1073-1079

95. Liu, K. et al. (2006) Shape-controlled production of biodegradable calcium alginate gel microparticles using a novel microfluidic device. Langmuir 22, 9453-9457

96. Zhang, $\mathrm{H}$. et al. (2006) Microfluidic production of biopolymer microcapsules with controlled morphology. J. Am. Chem. Soc $128,12205-12210$

97. Lin, Y.S. et al. (2013) Microfluidic synthesis of tail-shaped alginate microparticles using slow sedimentation. Electrophoresis 34, 425-431

98. Utech, S. et al. (2015) Microfluidic generation of monodis perse, structurally homogeneous alginate microgels for cell encapsulation and 3D cell culture. Adv. Healthc. Mater. 4 , 1628-1633

99. Prusse, U. et al. (2000) Production of spherical beads by JetCutting. Chem. Eng. Technol. 23, 1105-1110

100. Prusse, U. et al. (2008) Comparison of different technologies for alginate beads production. Chem. Paper 62, 364-374

101. Xu, T. et al. (2008) High-throughput production of single-cel microparticles using an inkjet printing technology. J. Manuf. Sci. Eng. - Trans. ASME 130, 021017

102. Nakamura, M. et al. (2005) Biocompatible inkjet printing technique for designed seeding of individual living cells. Tissue Eng. $11,1658-1666$

103. Wijshoff, H. (2010) The dynamics of the piezo inkjet printhead operation. Phys. Rep. 491, 77-177

104. Kwok, K.K. et al. (1991) Production of 5-15-Mu-M diamete alginate-polylysine microcapsules by an air-atomization technique. Pharm. Res. 8, 341-344 


\section{Trends in Biotechnology}

105. Cui, J.H. et al. (2001) Preparation and physical characterization of alginate microparticles using air atomization method. Drug Dev. Ind. Pharm. 27, 309-319

106. Strasdat, B. and Bunjes, H. (2013) Incorporation of lipid nanoparticles into calcium alginate beads and characterization of the encapsulated particles by differential scanning calorimetry. Food Hydrocoll. 30, 567-575

107. Hendriks, J. et al. (2015) Optimizing cell viability in droplet-based cell deposition. Sci. Rep. 5, 11304
108. Ganan-Calvo, A.M. et al. (1997) Current and droplet size in the electrospraying of liquids. Scaling laws. J. Aerosol Sci. 28, 249275

109. Nedović, V.A. et al. (2006) Optimization of the electrostatic droplet generation process for controlled microbead production: single nozzle system. Chem. Ind. Chem. Eng. Q. 12, 5

110. Jaworek, A. (2008) Electrostatic micro- and nanoencapsulation and electroemulsification: a brief review. J. Microencapsul. 25 $443-468$ 